Pacific Journal of Mathematic 


\title{
NATURALLY INTEGRABLE FUNCTIONS
}

\author{
Lester E. Dubins aNd David Margolies
}

A bounded function $f$ defined on an amenable group $G$ is naturally integrable if, for every pair of left-invariant means $\mu$ and $\mu^{\prime}, \mu(f)=\mu^{\prime}(f)$. If $G$ is the additive group of integers, then (i) $f$ is naturally integrable if, and only if,

$$
\lim n^{-1} \Sigma f(j+i)(1 \leqq i \leqq n)
$$

exists uniformly in $j$, and (ii) the associated natural measure $\nu$ is convex; that is, for every pair of naturally measurable sets of integers $E_{0}$ and $E_{1}$ with $E_{0} \subset E_{1}$, there is a monotone family of naturally measurable sets $E_{t}(0 \leqq t \leqq 1)$ such that $\nu\left(E_{t}\right)(0 \leqq t \leqq 1)$ is a closed interval. Analogous results hold for the presently known amenable groups.

An order-preserving linear functional $\mu$-or integral - defined on the space $B(G)$ of bounded, real-valued functions defined on a group $G$, is a (left)-invariant mean if $\mu(c)=c$ for all constants $c$ and if

$$
\mu f=\int f(x y) d \mu(y)
$$

for all $x \in G$; and $G$ is amenable if such a $\mu$ exists.

If $\mu f=\mu^{\prime} f$ for all invariant means $\mu$ and $\mu^{\prime}$, then $f$ is (left) naturally integrable. This section is concerned with characterizing the set, $\mathscr{N}$, of left naturally integrable functions. As a preliminary, a necessary and sufficient condition for $G$ to be amenable will be given.

For each finite subset $\alpha$ of $G$ and each $f \in B(G)$, the convolution of $\alpha$ with $f, \alpha * f$ is defined by

$$
(\alpha * f)(y)=\frac{1}{|\alpha|} \sum f(x y)(x \in \alpha)
$$

where $|\alpha|$ is the cardinality of $\alpha$. Plainly, for any invariant $\mu$,

$$
\mu f \leqq \overline{\alpha * f}, \text { for each } \alpha,
$$

where $\bar{f}$ is the supremum of $f$.

Summarizing,

$$
\mu f \leqq p(f)
$$

where

$$
p(f)=\inf _{\alpha} \overline{\alpha * f} .
$$


Of course, $p(t f)=t p(f)$ for $t>0$.

Proposition 1. For $G$ to be amenable, it is necessary and sufficient that $p$ be subadditive. If $p$ is subadditive then, for each $f \in B(G),-p(-f) \leqq p(f)$ and, for each $c$ in the closed interval, $[-p(-f), p(f)]$, there is a left-invariant $\mu$ for which $\mu(f)=c$. Moreover, there are no other values of $\mu(f)$ for left-invariant $\mu$.

For each nonempty, finite subset $\beta$ of $G$, let $\beta^{\prime}$ be the uniform distribution - or integral over $\beta$, defined thus:

$$
\beta^{\prime} f=\frac{1}{|\beta|} \sum f(y)(y \in \beta) .
$$

In terms of the total variation norm, \|\| , the necessary and sufficient condition of Følner ([8], [11]) for $G$ to be amenable can be stated, thus.

(F.C.) For every $\alpha$ and $\varepsilon>0$, there is a $\beta$ such that

$$
\left\|\beta^{\prime}-(x \beta)^{\prime}\right\|<\varepsilon \text { for all } x \in \alpha,
$$

where $x \beta$, the left translate of $\beta$ by $x$, is the set of all $x y, y \in \beta$. When (7) holds, $\beta$ is $\varepsilon$-invariant under $\alpha$.

Lemma 1. Suppose $G$ is amenable. Then, for every finite subset $\gamma$ of $G$ and $\varepsilon>0, \exists \beta \subset G$ such that, for all $\alpha \subset \gamma$ and all $f \in B(G)$ of absolute value at most 1 ,

$$
\left|\alpha^{*} \beta^{*} f-\beta^{*} f\right|<\varepsilon
$$

and, a fortiori,

$$
\overline{\beta * f}<\overline{\alpha * f}+\varepsilon .
$$

In fact, every $\beta$ which is $\varepsilon$-invariant under $\gamma$ satisfies (8) and (9).

Proof of Proposition 1. Suppose that $G$ is amenable, let $f_{1}$ and $f_{2}$ belong to $B(G)$, suppose $\left|f_{i}\right| \leqq 1$ and let $\varepsilon>0$. Then there are $\alpha_{1}$ and $\alpha_{2}$ such that $\overline{\alpha_{i}^{*} f_{i}}<p\left(f_{i}\right)+\varepsilon$. For $\gamma=\alpha_{1} \cup \alpha_{2}$ and $\beta$ chosen so that (9) holds, calculate, thus,

$$
\begin{aligned}
p\left(f_{1}+f_{2}\right) & \leqq \overline{\beta *\left(f_{1}+f_{2}\right)} \\
& \leqq \overline{\beta * f_{1}}+\overline{\beta * f_{2}} \\
& \leqq \overline{\alpha_{1}^{*} f_{1}}+\varepsilon+\overline{\alpha_{2}^{*} f_{2}}+\varepsilon \\
& \leqq p\left(f_{1}\right)+3 \varepsilon+p\left(f_{2}\right)+3 \varepsilon,
\end{aligned}
$$

which proves that the subadditivity of $p$ is a necessary condition 
for $G$ to be amenable. Suppose now that $p$ is subadditive. Then

$$
0=p(0)<p(-f+f) \leqq p(-f)+p(f),
$$

which proves that $-p(-f) \leqq p(f)$. That for each $f \in B(G)$ and each $c$ in the closed unit interval, there is a linear functional $\mu$ defined on $B(G)$ for which $\mu(f)=c$ and that each such $\mu$ is a left-invariant mean is established in almost direct imitation of Banach's argument for the existence of invariant means when $G$ is the additive group of the integers [1]. That no $c$ greater than $p(f)$ is a possible value of $\mu(f)$ for a left-invariant mean $\mu$ is the content of (4). And since

$$
\mu(f)=-\mu(-f) \text { and } \mu(-f) \leqq p(-f),
$$

$-p(-f)$ is a lower bound for $\mu(f)$.

Plainly, for each left-invariant mean $\mu, \mu$ annihilates the uniform closure $\kappa$ of the linear span, $\kappa_{0}$, of all functions of the form $f-L f$ where $L f$ is some left translate of $f$ and $f \in B(G)$. Therefore, the uniform closure of $R+\kappa_{0}$ is a subset of $\mathscr{N}$, where $R+\kappa_{0}$ designates the set of all functions of the form $c+f^{\prime}$ for $c$ a constant, that is, an element of $R$, and $f^{\prime} \in \kappa$. As demonstrated by Granirer and Witz in [9] and [21], there are no other elements of $\mathscr{N}$.

Three definitions facilitate the statement of the theorem. Of course, the oscillation of a real-valued function is the difference between its supremum and its infimum. If, for every $\varepsilon>0$, there is an $\alpha$ such that $\left|\alpha^{*} f-c\right|$ is at most $\varepsilon, f$ is averagable to the constant $c$. If, for some $n$, there exist $f_{1}, \cdots, f_{n}$ and $f_{1}^{\prime}, \cdots, f_{n}^{\prime}$ such that $f=\Sigma f_{i}, f^{\prime}=\Sigma f_{i}^{\prime}$ and, for each $i, f_{i}$ is a left translate of $f_{i}^{\prime}$, then $f$ and $f^{\prime}$ are (left) scissor-congruent. The following is at most than an embellishment of ideas and results in $[3,4,9,14,21]$.

Proposition 2. Suppose $G$ is amenable. Then the following conditions on a bounded $f$ are equivalent.

(a) $f$ is naturally integrable.

(b) $p(f)=-p(-f)$.

(c) $f$ is averageable to a constant.

(d) For every $\varepsilon>0$, there is a finite, nonempty subset $\alpha$ of $G$ such that the oscillation of $\alpha * f$ is at most $\varepsilon$.

(e) For every $\varepsilon>0$, there is an $f^{\prime} \in B(G)$ whose oscillation is at most $\varepsilon$ and which is scissor-congruent to $f$.

(f) $f$ is in the uniform closure of the linear space of those functions which are scissor-congruent to constant functions.

(g) $f$ is in the uniform closure of the set of functions of the form $c+\Sigma_{1}^{n}\left(f_{i}-L_{i} f_{i}\right)$ where $c$ is a constant and, for each $i, L_{i} f_{i}$ is a left translate of $f_{i} \in B(G)$. 
For the proof of Lemma 1, three lemmas are aseful.

Lemma 2. Suppose $G$ is amenable, $f \in B(G)$ and $\varepsilon>0$. Then, for some nonempty, finite subset $\beta$ of $G$,

$$
-p(-f)-\varepsilon<\beta * f<p(f)+\varepsilon .
$$

Proof. For some $\alpha_{1}$ and $\alpha_{2}$,

$$
\alpha_{1} * f<p(f)+\varepsilon / 2 \text { and } \alpha_{2} *(-f)<p(-f)+\varepsilon / 2 \text {. }
$$

Therefore, Lemma 1 yields a $\beta$ such that

$$
\beta * f<p(f)+\varepsilon \text { and } \beta *(-f)<p(-f)+\varepsilon,
$$

which implies (13).

Let $I(f)$ be the set of all numbers $c$ such that, for some leftinvariant $\mu, \mu(f)=c$.

LEMMA 3. Each of the following conditions implies its successors.

(i) For some finite, nonempty set $\alpha, f^{\prime}=\alpha * f$.

(ii) $f^{\prime}$ is left scissor-congruent to $f$.

(iii) $f-f^{\prime}$ belongs to $\kappa_{0}$.

(iv) For every left-invariant mean $\mu, \mu\left(f^{\prime}\right)=\mu(f)$.

(v) $I\left(f^{\prime}\right)=I(f)$.

LEMMA 4. The set of (left) naturally integrable functions is a uniformly closed, linear space.

Proof of Proposition 2. That (a) implies (b) is an evident corollary to Proposition 1. With the help of Lemma 2, the implication (b) $\rightarrow$ (c) is easily verified. That (c) implies (d) is trivial. The observation made in Lemma 3 that (i) $\rightarrow$ (ii) makes obvious the implication $(\mathrm{d}) \rightarrow(\mathrm{e})$. To see that (e) implies (f), proceed thus. Assume (e) and suppose: the oscillation of $f^{\prime}$ is at most $\varepsilon$ and $f^{\prime}$ is scissorcongruent to $f$. So $f=\sum_{1}^{n} f_{i}, f^{\prime}=\sum_{1}^{n} f_{i}^{\prime}$ and, for each $i, f_{i}=L_{i} f_{i}^{\prime}$ is a left translate of $f_{i}^{\prime}$. Let $\varepsilon^{\prime}$ be the difference between the supremum of $f^{\prime}$ and $f^{\prime}$, so $\varepsilon^{\prime}$ is a nonnegative function bounded above by $\varepsilon$. Let $f_{i}^{\prime \prime}=f_{i}^{\prime}+(1 / n) \varepsilon^{\prime}$. Plainly, $\Sigma f_{i}^{\prime \prime} \equiv c$, the supremum of $f^{\prime}$. Now calculate, thus

$$
\begin{aligned}
0 & \leqq \Sigma L_{i} f_{i}^{\prime \prime}-f \\
& =\Sigma L_{i}\left(f_{i}^{\prime}+\frac{1}{n} \varepsilon^{\prime}\right)-\Sigma L_{i} f_{i}^{\prime}
\end{aligned}
$$




$$
\begin{aligned}
& =\frac{1}{n} \Sigma L_{i} \varepsilon^{\prime} \\
& \leqq \varepsilon .
\end{aligned}
$$

So $f$ differs from $\Sigma L_{i} f_{i}^{\prime \prime}$, a function scissor-congruent to a constant function, by at most $\varepsilon$. This proves that $(\mathrm{e}) \rightarrow(\mathrm{f})$. Suppose $(f)$ holds. Then for each $\varepsilon>0$, there is an $f^{\prime}$ such that $\left|f-f^{\prime}\right|<\varepsilon$ and, for some constant $c, f^{\prime}$ is scissor-congruent to $c$. So there exist $f_{1}^{\prime}, \cdots, f_{n}^{\prime}$ such that $\Sigma f_{1}^{\prime}=f^{\prime}$ and $\Sigma L_{i} f_{i}^{\prime}=c$ where $L_{i} f_{i}^{\prime}$ is some left translate of $f_{i}$. So $f^{\prime}-c=\Sigma f_{i}^{\prime}-L_{i} f_{3}^{\prime}$ belongs to $\kappa_{0}$, or equivalently, $f^{\prime} \in R+\kappa_{0}$. Consequently, (g) holds. That (g) implies (a) is evident from Lemma 4 .

The remainder of this section is concerned with two applications. The first is a generalization, as well as a slight strengthening of, Hermann Weyl's observation that if $\theta$ is an irrational real number, then the sequence $n \theta$ taken modulo 1 is equidistributed. The second states - in a sense stronger than usual - that the primes are a thin subset of the integers.

If, for every $\varepsilon>0$, there are continuous functions $w^{\prime}$ and $w^{\prime \prime}$ on a compact group such that $w^{\prime}<w<w^{\prime \prime}$ and such that the Haar integral of $w^{\prime \prime}-w^{\prime}$ is less than $\varepsilon$, then $w$ is Eudoxian integrable.

Proposition 3. Let $\varphi$ be an isomorphism of a group $G$ onto a dense subgroup of a compact group $K$. Let $w$ be a Eudoxian integrable function defined on $K$ and let $f=w \circ \varphi$ be the composition of $w$ with $\varphi$. Then $f$ is averagable to a unique constant. Consequently, if $G$ is amenable, $f$ is naturally integrable.

Proof. Let $\varepsilon>0$ and let $w^{\prime}$ and $w^{\prime \prime}$ be continuous, real-valued functions defined on $G$ such that $w^{\prime}<w<w^{\prime \prime}$ and such that $\lambda w^{\prime \prime}$ $\lambda w^{\prime}<\varepsilon$ where $\lambda$ is the Haar integral. As Von Neumann's ([16], [17]) development of the Haar integral for compact groups makes evident, there is a finite subset $\alpha$ of $G$ such that $\left|\alpha * w^{\prime}-\lambda w^{\prime}\right|$ and $\left|\alpha * w^{\prime \prime}-\lambda w^{\prime \prime}\right|$ are everywhere less than $\varepsilon$. Since $\varphi(G)$ is dense in $K$, it may be assumed that $\alpha$ is a subset of $\varphi(G)$. Moreover, since $\alpha * w$ is between $\alpha * w^{\prime}$ and $\alpha * w^{\prime \prime}$, an evident calculation shows that $|\alpha * w-\lambda w|$ is at most $2 \varepsilon$. Let $\beta$ be the subset of $G$ which $\varphi$ maps onto $\alpha$. It is now clear that $|\beta * f-\lambda w|$ is at most $2 \varepsilon$ and, therefore, that $f$ is averageable to the constant $\lambda w$. Finally, if $G$ is amenable, Proposition 2 implies that $f$ is naturally integrable.

Incidentally, whether $G$ is amenable or not, the conclusion of Proposition 1 could be strengthened to say: for any $\varepsilon>0$ and any 
finite set $W$ of Eudoxian integrable functions defined on $K$, there is a finite subset $\beta$ of $G$ such that, for all $w \in W,|\beta *(w \circ \varphi)-\lambda w|<\varepsilon$ everywhere on $G$.

EXAmple 1. Let $A$ be an arc of the unit circle $Z$ and suppose $z \in Z$ is not a root of unity. Then the set $S=S_{A, z}$ of integers $n$ for which $z^{n} \in A$ is a naturally measurable set of integers whose measure, $\rho$, is proportional to the length of $A$. That $S$ has a density equal to $\rho$ is a well-known observation of Hermann Weyl's ([19], [20]).

As a prelude to the next application, namely Proposition 4, the following obvious fact is recorded here.

Lemma 5. The infinite cyclic group generated by a positive integer $n$, as well as each of its translates, has measure $1 / n$ under. every translation-invariant measure defined on the integers.

Another example of a naturally measurable set of integers is the set of primes. The following argument is due to Nathan Fine who explained it in conversation with one of us over twenty years ago.

Lemma 6. For every $\varepsilon>0$, there is a positive integer $n$ such that fewer than ne translates of the infinite cyclic group $H_{n}$ generated by $n$ cover the set of primes.

Proof. As is well-known, there is a positive integer $m$ such that $\varphi(m)$, the cardinality of the set, $S$, of positive integers $i<m$ which are relatively prime to $m$, is less than $m \varepsilon / 2$. (See, for example, [12, Theorems 19 and 60].) As is not difficult to verify, every positive integral multiple of $m$ which exceeds $2 m / \varepsilon$ is an $n$ which satisfies the lemma.

Proposition 4. There is one, and only one, translation-invariant probability measure defined on the smallest translation-invariant field which includes the arithmetic sequences. With respect to the completion of this measure, the set of primes is measurable and has measure zero; a fortiori, the set of primes is naturally measurable and has natural measure zero.

Proof. Immediate from Lemmas 5 and 6.

2. Convexity of invariant means. This section extends certain results of Granirer [10] and of Chou [2] by showing that each invariant mean, $\mu$, on an infinite, amenable group, $G$, possesses a certain property enjoyed by nonatomic, countably additive measures 
defined on sigma-fields, namely: For each $A \subset G$, for $B$ ranging over the subsets of $A$, the range of values of $\mu(B)$ is a closed interval.

We begin by presenting formally certain ideas of de Finetti [6, p. 117]. A collection of sets which has the property that every set which is a finite, linear combination of sets of the collection belongs to the collection is a linear space of sets. Here, the useful convention, also introduced by de Finetti [2] of identifying a set with its indicator function is being adopted. In this paper, it will always be assumed that the universal set $\Omega$ or, equivalently, the constant function 1 , is an element of the linear space.

The probability measures defined on a linear space, $\mathscr{L}$, of events is plainly a convex set whose extreme points are the two-valued, or zero-one, probabilities, that is, those that assign to every $E \in \mathscr{L}$ the value of zero or one. As usual, if $\mu$ and $\mu^{\prime}$ are measures such that $\mu E \geqq \mu^{\prime} E$ for all $E \in \mathscr{L}$, then $\mu$ is said to majorize $\mu^{\prime}$ or, more concisely, $\mu \geqq \mu^{\prime}$.

If, whenever an index $s$ is less than an index $t, E_{s} \leqq E_{t}$, the family $\left\{E_{t}\right\}$ is a chain.

Lemma 1. The following conditions on a probability $\mu$ defined on a linear space $\mathscr{L}$ of events are equivalent.

(a) For every extreme probability $\mu^{\prime}$ and real number $c$, if $\mu \geqq c \mu^{\prime}$, then $c \leqq 0$.

(b) For all $E \in \mathscr{L}$, with $\mu(E)>0$ and all $\varepsilon>0$, there is an $E_{1} \in \mathscr{L}, E_{1} \subset E$ such that

$$
\frac{1}{2}-\varepsilon<\frac{\mu\left(E_{1}\right)}{\mu(E)}<\frac{1}{2}+\varepsilon .
$$

(c) For each $E \in \mathscr{L}$ and $\varepsilon>0$, there is a chain $\left\{E_{t}, t \in T\right\}$ which satisfies: $T$ is a finite subset of $[0,1]$ such that, for each $t^{\prime} \in(0,1)$, there is a $t \in T$ with $\left|t-t^{\prime}\right|<\varepsilon ; E_{t} \in \mathscr{L}$ and $\mu\left(E_{t}\right)=t$ for all $t \in T$; for some $t \in T, E_{t}=E$.

(d) For each $E \in \mathscr{L}$, there is a chain $\left\{E_{t}, t \in T\right\}$ such that $T$ is $a$ dense subset of $[0,1], E_{t} \in \mathscr{L}$ and $\mu E_{t}=t$ for all $t \in T$ and, for some $t \in T, E_{t}=E$.

In the event $\mathscr{L}$ is a field of subsets of a set $\Omega$, for (c) or (d) to hold for all $E$ it suffices that it holds for $E=\Omega$.

For a probability $\mu$ with a given domain, $\mathscr{L}$, the outer $\mu$ probability, $\mu^{*}$ or $\mu_{\mathscr{S}}^{*}$ is defined, as usual, for every event $E$ as the infimum of $\mu\left(E^{\prime}\right)$ for $E^{\prime} \in \mathscr{L}, E \subset E^{\prime}$. With the inner $\mu$-probability analogously defined, call $(\mu, \mathscr{L})$ order-complete if every $E$ with equal inner and outer $\mu$-probability is an element of $\mathscr{L}$. When $\mu$ is 
understood, $\mathscr{L}$ is called order-complete, and when $\mathscr{L}$ is understood, it is $\mu$ which is so labelled.

LeMma 2. If $(\mathscr{P}, \mu)$ is order-complete, this condition, too, is equivalent to (d).

(d') For every $E \in \mathscr{L}$ and every $t$ in the interval $[0,1]$, there is an $E_{t} \in \mathscr{L}$ such that $E_{t}=t, s \leqq t \rightarrow E_{s} \subset E_{t}$ and, for some $t, E_{t}$ is $E$.

If (a), (b), (d) or (d) of Lemma 1 holds, $\mu$ is continuous; if (d') holds, $\mu$ is convex.

LEMMA 3. If $\mu$ is a probability measure defined on a linear space $\mathscr{L}$ of events then, for each positive integer $n$, there are at most $n$ distinct two-valued probabilities $\mu^{\prime}$ defined on $\mathscr{L}$ such that " majorizes $(1 / n) \ell^{\prime}$.

Proof. As is easily verified, distinct two-valued probabilities are mutually singular. Therefore if $\mu$ majorizes $(1 / n) \mu_{j}^{\prime}$ for $j$ in a finite set, then $\mu$ majorizes $(1 / n) \Sigma \mu_{j}^{\prime}$, from which it follows that the index set has at most $n$ members.

Recall that if $\iota^{\prime}$ is any probability on a group $G$ and $g \in G$, then $g \ell^{\prime}$ is the left translate of $\mu^{\prime}$ by $g$. Of course, if $\mu^{\prime}$ is two-valued, so is $g \ell^{\prime}$.

Lemma 4. It $\mu^{\prime}$ is a two-valued probability on group $G$, then the $\operatorname{map} g \rightarrow g \mu^{\prime}$ is injective.

Proof. It plainly suffices to show that, unless $g$ is the identity, $e$ of $G, g \mu^{\prime}$ and $\mu^{\prime}$ are distinct. As will soon be shown, for each $g \neq e$, there is a subset $A$ of $G$ and an integer $n \geqq 2$ such that $A$, $g A, \cdots, g^{n-1} A$ constitute a $g$-cyclic partition $\pi$ of $G$, that is, one for which $g^{n} A=A$. Since one and only one of these sets, say $g^{i} A$, has $\ell^{\prime}$ measure 1 , its successor $g^{i+1} A$ has $\mu^{\prime}$-measure 1 under $g \mu^{\prime}$, and hence $g \ell^{\prime}$ is certainly distinct from $\mu^{\prime}$. To verify the existence of the partition $\pi$, let $H$ be the group generated by $g$ and let $L \subset G$ contain one and only one element from each right coset of $H$. If $H$ is finite, let $A=L$, so $n$ is the order of $H$; if $H$ is infinite, let $S$ be the group generated by $g^{2}$, and let $A=S L$, so $n=2$. In either case, $A$ generates a $g$-cyclic partition of $G$, as is easily verified.

Proposition 1. Every invariant mean on an infinite amenable group is convex. 
Proof. Let $\mu$ be an invariant mean for an infinite amenable group $G$, let $\mu^{\prime}$ be an extreme probability and suppose that $\mu \geqq(1 / n) \mu^{\prime}$ for some positive integer $n$. Then $\mu=g \mu \geqq(1 / n) g \mu^{\prime}$ for all $g \in G$. As Lemma 4 now implies, $\mu \geqq(1 / n) \mu^{\prime \prime}$ for infinitely many different extreme probabilities $\mu^{\prime \prime}$. But this is impossible according to Lemma 3. So $\mu$ satisfies (a) and, consequently, (d) of Lemma 1. Since $\mu$ is defined for all subsets of $G$, it is trivially order-complete, so Lemma 2 now implies that $\mu$ satisfies $\left(d^{\prime}\right)$, that is, $\mu$ is convex.

3. Naturally measurable sets of integers. The following variant of a result of Jerison [13] which is generalized in the next section finds application below.

Proposition 1. For an $f$ defined on the infinite cycle group of integers to be naturally integrable it is necessary and sufficient that

$$
\lim n^{-1} \sum f(j+i)(1 \leqq i \leqq n)
$$

exists uniformly in $j$.

Proof. Since a function which satisfies the condition is plainly a verageable to a constant, Theorem 1 of $\S 1$ yields the sufficiency of the condition. For the necessity, let $f$ be naturally integrable with integral $c$, suppose $|f| \leqq 1$, and let $\varepsilon$ be positive. By Theorem 1 , of Section 1, there is an $\alpha$ such that $\alpha * f$ has all of its values in the interval $[c-\varepsilon, c+\varepsilon]$. Choose $n$ sufficiently large so that the finite set $\beta=\{1, \cdots, n\}$ is $\varepsilon$-invariant under $\alpha$. By Lemma 1 of Section $1, \beta * f$ has all its values in the interval $[c-2 \varepsilon, c+2 \varepsilon]$. Since $\beta * f$ is the function whose value at $j$ is $n^{-1} \Sigma f(j+i)(1 \leqq i \leqq n)$, the proposition is proved.

In contrast to the set of primes, the set $S$ of square free integers is not naturally measurable. For, on the one hand, $S$ has a nonzero density, namely $6 / \pi^{2}$, as is well-known (see e.g., [12, Theorem 333].) On the other hand, as will soon be verified, for $n$, there are $n$ successive integers none of which belong to $S$. This implies, in view of Proposition 1, that, were $S$ naturally measurable, its natural measure would be zero. So to complete the proof of the above italicized statement, only this lemma need be established.

LEMMA 1. For every positive integer $n$, there are $n$ successive positive integers each of which has a nontrivial square divisor. Indeed, for any $n$ distinct primes $p_{1}, \cdots, p_{n}$, there is a positive integer $j$ such that $j+i$ is divisible by $p_{i}^{2}, 1 \leqq i \leqq n$. 
Proof. That the system of $n$ congruences $j+i \equiv 0 \bmod p_{i}^{2}, 1 \leqq$ $i \leqq n$, has a solution is an immediate consequence of the Chinese Remainder Theorem. (See, for example, [12, Theorem 121].)

4. Convexity of natural measures. The theorem of this section says that if $G$ is any member of a well-known class of amenable groups - possibly all - then its (left) natural measure is convex. For earlier related results, see [2], [10] and [8].

The (left) natural measure of an amenable group $G$ is simply the restriction of any left invariant mean on $G$ to the collection of (left) naturally measurable subsets of $G$.

If $H$ is a normal subgroup of $G$ and $G / H$ is isomorphic to $Q$, then $G$ is an extension of $Q$ by $H$. If $\mathscr{G}$ is a collection of groups such that $H \in \mathscr{G}$ and $Q \in \mathscr{G}$ implies that $\mathscr{G}$ contains every extension of $Q$ by $H$, then $\mathscr{G}$ is closed under extension.

Call $\mathscr{G}$ directed if, for every $\left(G_{1}, G_{2}\right)$ with $G_{i} \in \mathscr{G}, i=1,2$, there is a $G \in \mathscr{C}$ which includes both $G_{1}$ and $G_{2}$ as subgroups. If $\mathscr{G}$ is directed, then $V \mathscr{G}$, the set-theoretic union of all $G \in \mathscr{G}$, is obviously a group. If, for every directed subset $\mathscr{C}$ of $\mathscr{G}, V \mathscr{C} \in \mathscr{G}$, then is closed under directed limits.

Call a group $G$ accessible, and write $G \in \mathscr{A}$, if $G \in \mathscr{G}$ whenever $\mathscr{G}$ satisfies this condition: $\mathscr{G}$ is closed under both group extension and directed limits and $\mathscr{C}$ includes all finite groups as well as the infinite cyclic groups. As is not difficult to verify, solvable groups are accessible.

Theorem 1. If $G$ is an infinite, accessible group, then its natural measure is convex.

The proof of Theorem 1 requires the introduction of a class of groups which is shown to include $\mathscr{A}$, the class of infinite, accessible groups, and to be included in $\mathscr{C}$, the class of amenable groups whose natural measure is convex.

A tiling $\tau$ of $G$ is an ordered pair $(\beta, J)$ where $\beta$ and $J$ are nonempty subsets of $G$ such that the set of all right-translates of $\beta$ by elements of $J$, or equivalently, the set of all left-translates of $J$ by elements of $\beta$, constitutes a partition of $G$. If $|\beta|<\infty, \tau$ is proper.

A collection of proper tilings of $G$ is adequate if, for all finite subsets $\alpha$ of $G$ and $\varepsilon>0$, there is a tiling $(\beta, J)$ in the collection such that $\beta$ is $\varepsilon$-left invariant under $\alpha$.

LEMMA 1. Every accessible group possesses an adequate collection of proper tilings. 
Proof. Consider first the trivial case of a finite group, G. For any $e \in G,(G,(e))$ is a tiling of $G$, and this one-point set of tilings is plainly adequate. Now consider $G$, the infinite cyclic group of integers. For $n \in G, n>1$, let $J=n G$ be the cyclic subgroup generated by $n$ and let $\beta$ be the set of $n$ successive integers $1, \cdots, n$. As is easily verified, especially by reference to Proposition 1 of Section 3, $(\beta, J)$ is a proper tiling of $G$ and the set of such tilings is adequate.

Assume next that $H$ is a normal subgroup of a group $G$, that $H$ and $G / H$ belong to the set, $\mathscr{B}$, of groups which possess adequate collections of proper tilings. To see that $G \in \mathscr{B}$, one may proceed, thus. Let $D$ be a finite subset of $G$ and let $\varepsilon>0$. Plainly, if $\pi$ is the canonical projection of $G$ onto $G / H, \pi D$ is a finite subset of $G / H$. Since $G / H \in \mathscr{B}$, there is a tiling $\left(\beta^{\prime}, I^{\prime}\right)$ of $G / H$ which is $\varepsilon$-invariant under $\pi D$. Let $\beta$ be a selection from $\beta^{\prime}$, that is, a subset of $G$ with the property that $\pi$ is a one-to-one map of $\beta$ onto $\beta^{\prime}$. Plainly, $\beta^{-1} D \beta$ and, a fortiori, the intersection of $H$ with $\beta^{-1} D \beta$, is a finite set, where $\beta^{-1}$ designates, of course, the set of the inverses of the elements of $\beta$. Since $H \in \mathscr{B}$, there is a tiling $(\alpha, J)$ of $H$ which is $\varepsilon$-invariant under $H \cap\left(\beta^{-1} D \beta\right)$. Now let $I$ be an $I^{\prime}$-selection, that is, a subset of $G$ with the property that $\pi$ is a one-to-one map of $I$ onto $I^{\prime}$. As is easily verified, $(\beta \alpha, J I)$ is a tiling of $G$. There remains, therefore, only to verify that $\beta \alpha$ is $(2 \varepsilon)$-invariant under $D$. For this purpose, note first that $|\beta \alpha|$, the cardinality of $\beta \alpha$, is simply the product of $|\beta|$ by $|\alpha|$. Then fix $d \in D$ and let $\beta_{d}$ be that subset of $\beta$ consisting of all $b^{*}$ such that $d b^{*}=b h$ for some $b \in \beta$ and some $h \in H$. Note next that $\left|\beta_{d}\right|>(1-\varepsilon)|\beta|$, for $\beta^{\prime}$ is $\varepsilon$-invariant under $d H$. Now fix $b^{*} \in \beta_{d}$, so the relation $d b^{*}=b h$ determines unique $b \in \beta$ and $h \in H$, and let $\alpha^{*}$ be the set of $a \in \alpha$ such that $h a \in \alpha$. Since $\alpha$ is $\varepsilon$-invariant under $h,\left|\alpha^{*}\right|>(1-\varepsilon)|\alpha|$. Recapitulating, for $b^{*} \in \beta_{d}$ and $a \in \alpha^{*}, d b^{*} a=b h a=b a^{\prime} \in \beta \alpha$. As the map $\left(b^{*}, a\right) \rightarrow b^{*}$ $a$ from $\beta \times \alpha \rightarrow G$ is easily verified to be one-to-one, the set of $b^{*} a$, $b^{*} \in \alpha, a \in \alpha$ such that $d b^{*} a \in \beta \alpha$ has cardinality at least $(1-\varepsilon)^{2}|\beta||\alpha|$ which exceeds $(1-2 \varepsilon)|\beta \alpha|$. So $\beta \alpha$ is $2 \varepsilon$-invariant under $D$, which completes the proof that $G \in \mathscr{B}$.

Let $G=V \mathscr{C}$ where $\mathscr{H}$ is a directed subset of $\mathscr{G}$. Let $\varepsilon>0$ and let $\alpha$ be a finite subset of $G$. Plainly, for some $H \in \mathscr{H}, \alpha \subset H$. So, there is a tiling $(\beta, J)$ of $H$ which is $\varepsilon$-invariant under $\alpha$. The axiom of choice implies the existence of a subset $K$ of $G$ such that $(H, K)$ is a tiling of $G$. As is now easily verified, $(\beta, J K)$ is a thing of $G$ which is, of course, $\varepsilon$-invariant under $\alpha$. Consequently, $G \in \mathscr{B}$. So $\mathscr{B}$ is closed under directed limits.

[Incidentally, in view of Lemma 1, if it should prove possible to construct a group which, on the one hand, satisfies Følner's condition 
but, on the other, does not possess an adequate set of tilings, a 'new' amenable group would thereby be obtained. For the only discrete amenable groups which seem to be presently known are accessible.]

Proper tilings generate naturally measurable functions and sets, as the next lemma suggests. If $\tau=(\beta, J)$ is a proper tiling of $G$, define $E(f \mid \tau)$, the conditional expectation of $f$ given $\tau$, to be that function $f^{*}$ defined on $G$ such that, for each $j \in J, f^{*}$ is constant on $\beta j$ and satisfies

$$
\Sigma f^{*}(g)(g \in \beta j)=\Sigma f(g)(g \in \beta j) .
$$

Lemma 2. For any $f$ and proper tiling $\tau, f$ is scissor-congruent to $E(f \mid \tau)$. Consequently, if $f$ is a bounded function defined on an amenable group $G$ and $E(f \mid \tau)$ is a constant, then $f$ is naturally integrable.

Proof. Consider first the special case that, for some $b \in \beta, f$ is zero except possibly on $b J$. For each $a \in \beta$, let $f^{a}$ be zero everywhere except on $a J$ where it agrees with $E(f \mid \tau)$. Plainly, $f^{a}$ is a left translate of $|\beta|^{-1} f$, so $E(f \mid \tau)$, the sum of the $f^{a}$, is scissor-congruent to $f$. For the general $f$, express $f$ as the sum of functions $f_{b}, b \in \beta$, where $f_{b}$ agrees with $f$ or $b J$ and is zero everywhere else. By the special case already considered, $f_{b}$ is scissor-congruent to $E\left(f_{b} \mid \tau\right)$. Since scissor-congruence is preserved under addition, $f$, the sum of the $f_{b}$, is scissor-congruent to $E(f \mid \tau)$, the sum of the $E\left(f_{b} \mid \tau\right)$.

LeMMA 3. If $G$ is an infinite, amenable group which possesses an adequate collection of proper tilings, then its natural measure is convex.

Proof. Plainly, for any amenable group the collection $\mathscr{C}$ of its naturally measurable subsets is a linear space of sets on which its natural measure $\nu$ is order-complete. So, in view of Lemma 2 of $\S 2$, it will suffice to show that every naturally measurable subset $A$ of $G$ can be split into two naturally measurable sets of almost equal measure. With this aim in view, let $f$ be the indicator function $A$ and let $\delta$ be a small positive number. As Proposition 2 of $\S 1$ says, for some finite subset $\alpha$ of $G$,

$$
\operatorname{osc}\left(\alpha^{*} f\right)<\delta .
$$

As will next be shown, the cardinality of $\alpha$ can be chosen arbitrarily large. For, if not, choose an $\alpha$ of maximal cardinality such that (2) obtains, and note first that for each $g \in G, \alpha g$, the right-translate of $\alpha$ by $g$, is another $\alpha$ which satisfies (2). Moreover, since $G$ is infinite, 
$g$ may be so chosen that $\alpha g$ is disjoint from $\alpha$. Plainly, $\alpha \cup \alpha g$ satisfies (2), which contradicts the assumed maximality of the cardinality of $\alpha$.

By hypothesis, there is a tiling $\tau=(\beta, J)$ such that $\beta$ is $\delta$ invariant under $\alpha$. Consider the largest integer $r$ such that, for every $j \in J,|A \cap(\beta j)| \geqq 2 r$, and let $B$ be a subset of $A$ such that, for every $j \in J,|B \cap(B j)|=r$. In view of Lemma $2, B$ is naturally measurable with measure $r / m$ where $m$ is the cardinality of $\beta$. Plainly, $\nu(B)=$ $r / m \leqq \nu(A) / 2$. There remains therefore only to verify that if $|\alpha|$, the cardinality of $\alpha$, is sufficiently large, then $\nu(A) / 2 \leqq \nu(B)+2 \delta$. For this purpose, notice first that for some $j, 2(r+1)>|A \cap \beta j|$, or, equivalently, that

$$
2(r+1) / m>(\beta * f)(j) \text { for some } j \in J .
$$

Since $\beta$ is $\delta$-invariant under $\alpha$, “ $\alpha * \beta$ " can of course be substituted for ' $\alpha$ ' in 2. So Lemma 1 of $\S 1$ implies that the oscillation of $\beta * f$ is less than $2 \delta$. This certainly entails that $\beta * f$ is everwhere greater than its mean minus $2 \delta$. This fact, together with (3), implies

$$
2(r+1) / m>\nu(A)-2 \delta,
$$

for the mean of $\beta * f$ is simply $\nu(A)$. Recapitulating,

$$
\begin{aligned}
\nu(A) / 2 & <(r+1) / m+\delta \\
& =\nu(B)+\delta+1 / m .
\end{aligned}
$$

So, to complete the proof of the lemma, there remains only to verify that if $|\alpha|$ is sufficiently large then $1 / m<\delta$, that is, that $1<\delta|\beta|$. But, because $\beta$ is $\delta$-invariant under $\alpha$, this last inequality is automatic provided that $1<\delta|\alpha|$, as is easily verified, thus. In the trivial case in which $|\beta| \geqq|\alpha|$, the conclusion is evident. In the remaining case, $|\beta|<|\alpha|$. In this case, for each $b \in \beta$, there is some $a \in \alpha$ such that $a b$ is not an element of $\beta$. But $\beta$ is $\delta$-invariant under $\alpha$. So $\delta>$ $1 /|\beta|$.

Plainly, Lemmas 1 and 3 together imply Theorem 1.

5. Two remarks. As has been noted, the naturally measurable subsets of an amenable group form a linear space of sets. Therefore, they are closed under disjoint unions, complements and proper differences. But they are not closed under intersections, as the following example shows. If $A \subset Z$ is not naturally measurable, then neither is $2 A$. Define $C=\{2 n+1: n \notin A, n \in Z\}$. If $D=(2 A) \cup C, D$ is easily seen to be naturally measurable for, if $\tau=(\{0,1\}, 2 Z), E(D \mid \pi) \equiv 1 / 2$. But neither $D \cap 2 Z$ nor $D \cup 2 Z$ is naturally measurable.

If a function is measurable with respect to the naturally mea- 
surable sets, it is, of course, naturally integrable. But the class of such functions is a proper subset of the class of naturally integrable functions. For if $g$ is any bounded, nonnegative function supported by the even integers, then $g-g_{1}$ is naturally integrable but is not measurable unless $g$ is.

\section{REFERENCES}

1. S. Banach, Sur ce probleme de measure, Fund. Math., 4 (1923), 7-33.

2. C. Chou, On a conjecture of $E$. Granirer concerning the range of an invariant mean, Proc. Amer. Math. Soc., 26 (1970), 105-107.

3. M. Day, Semigroups and amenability, in Semigroups, (Proc. Symp., Wayne State U.), Karl Folley ed., Academic Press, New York, 1969, 5-50.

4. — Amenable semigroups, Illinois J. Math., 1 (1957), 509-544.

5. L. Dubins, Naturally integrable functions, unpublished manuscript, University of California, Berkeley, 1977.

6. B. de Finetti, Probability, Induction, and Statistics, Wiley, New York, 1972.

7. - Theory of Probability, Vol. 1, Wiley, 1974.

8. E. Følner, On groups with full Banach mean value, Math. Scand., 3 (1955), 243-254.

9. E. Granirer, On left amenable semigroups which admit countable left invariant means $I$ and II, Illinois J. Math., 7 (1963), 32-48, 49-58.

10. On the range of an invariant mean, Trans. Amer. Math. Soc., 125 (1966), 384-394.

11. F. P. Greenleaf, Invariant Means on Topological Groups, Van Nostrand, 1969.

12. G. H. Hardy and E. Wright, An Introduction to the Theory of Numbers, 4th ed., Clarendon Press, Oxford, 1960.

13. M. Jerison, The set of all generalized limits of bounded sequences, Canad. J. Math., 9 (1957), 79-89.

14. G. Lorentz, $A$ contribution to the theory of divergent sequences, Acta Mathematica, 80 (1948), $167-190$.

15. J. von Neumann, Zur allgemeinen Theorie des Masses, Fund. Math., 13 (1929), $73-116$.

16. — Zum Haarschen Mass in topologischen Gruppen, Comp. Math., 1 (1934).

17. L. Pontriagin, Topological Groups, Princeton University Press, Princeton, 1946.

18. R. Snell, The range of invariant means on locally compact abelian groups, Canad. Math. Bull., 17 (1974), 567-573.

19. H. Weyl, Nachr. Akad. Wiss. Göttingen, (1914), 235-236.

20. - Math. Ann., 77 (1916), 313-315.

21. K. Witz, Applications of a compactification for bounded operator semigroups, Illinois J. Math., 8 685-696.

Received December 21, 1978. Research sponsored by National Science Foundation Grant No. MCS 75-01665.

This article is a fusion of a portion of the thesis of one of us (D. M.) with prior unpublished work (of L. E. D.). The remainder of D. M.'s thesis, which he submitted in partial fulfillment of the requirements of the $\mathrm{Ph}$. D. degree at U. C. Berkeley, treats of unrelated matters and D. M. plans to publish that portion separately.

Thanks are due to Aryeh Dvoretzky for a helpful comment he made to one of us in 1964 in Jerusalem and to Harry Furstenburg for one he made to both of us in 1977 in Berkeley.

UNIVERSITY OF CALIFORNIA

BERKELEY, CA 94720

LAWRENCE LIVERMORE NATIONAL LABORATORY

P. O. Box 808

LIVERMORE, CA 94550 
.././. ./FrontMatter/paper .pdf 


\section{Pacific Journal of Mathematics \\ Vol. 87, No. $2 \quad$ February, 1980}

Theagenis Abatzoglou, Unique best approximation from a $C^{2}$-manifold in Hilbert space ................................. 233

Gerald Arthur Anderson, $\Lambda$-homology cobordism bundles............. 245

Eric Bedford, Holomorphic mapping of products of annuli in $\mathbf{C}^{n} \ldots \ldots \ldots 271$

Gunnar Carlsson, On the stable splitting of $b o \wedge b o$ and torsion operations in connective $K$-theory .......................... 283

Lester Eli Dubins and David Samuel McIntyre Margolies, Naturally integrable functions ................................. 299

Leo Egghe, The Radon-Nikodým property, $\sigma$-dentability and martingales in locally convex spaces ............................. 313

Irving Leonard Glicksberg, Maps preserving translates of a function ..... 323

Hugh M. Hilden and Robert D. Little, Cobordism of branched covering

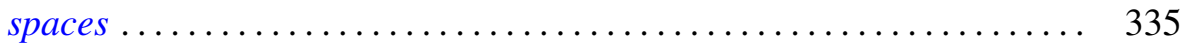

Russell Allan Johnson, Almost-periodic functions with unbounded integral .......................................... 347

Bruce Stephen Lund, The endomorphisms of a Dirichlet algebra ........ 363

John Henry McCleary, Mod $p$ decompositions of H-spaces; another approach........................................ 373

Arlan Bruce Ramsay, Subobjects of virtual groups ................ 389

Thomas R. Savage, Generalized inverses in regular rings ............ 455 Jaak Vilms, On curvature operators of bounded rank ......... 